\title{
Torsión del cordón espermático en un semental porcino
}

\author{
Sperm cord twist in a porcine stald
}

Reyes-Bossa BJ, Henriquez-Crespo RJ, Cardona-Álvarez JÁ. Torsión del cordón espermático en un semental porcino. Rev Colombiana Cienc Anim. Recia. 2020; 12(2):e786. https://doi.org/10.24188/ recia.v12.n2.2020.786

Universidad de Sucre, Colombia

Los autores permiten a RECIA reimprimir el material publicado en él. En caso de que un autor quiera traducir o usar una publicación parcial o completa de nuestro Diario, el autor debe obtener un permiso por escrito del editor de la revista.

Copyright (C) 2020. El (los) autor (es), Revista Colombiana de Ciencia Animal - RECIA. 2020. Este es un artículo de acceso abierto distribuido bajo los términos de Creative Commons Attribution 4.0 (https://creativecommons.org/licenses/by-nc-sa/4.0/), El uso, distribución o reproducción está permitido, siempre que se acrediten al autor original y al propietario del copyright y que se cite la publicación original en esta revista, de acuerdo con la práctica académica aceptada. No se permite el uso, distribución o reproducción que no cumpla con estos términos. 


\title{
Torsión del cordón espermático en un semental porcino
}

\author{
Sperm cord twist in a porcine stald
}

Bernardo José Reyes Bossa. M.Sc.

Universidad de Córdoba, Facultad de Medicina Veterinaria y Zootecnia, Grupo de Investigación en Medicina de Grandes Animales (MEGA), Montería, Colombia

breyesbossa@correo.unicordoba.edu.co

(iD) https://orcid.org/0000-0002-8730-0350

\section{Ricardo José Henriquez Crespo. M.Sc.}

Universidad de Córdoba, Facultad de Medicina Veterinaria y Zootecnia, Estudiante de Maestría en Ciencias Veterinarias del Trópico, Grupo de Investigación en Medicina de Grandes Animales (MEGA), Montería, Colombia

riyumix@hotmail.com

(iD https://orcid.org/0000-0001-9296-1666

José Alberto Cardona Álvarez. Dr.Vet.Sc.

Universidad de Córdoba, Departamento de Ciencias Pecuarias, Grupo de Investigaciones en Medicina de Grandes Animales (MEGA), Profesor Titular de Medicina y Clínica de Grandes Animales, Montería, Colombia. jacardonaalvarez@correo.unicordoba.edu.co

(iD) https://orcid.org/0000-0002-8254-1120
DOI: https://doi.org/10.24188/recia.v12.n2.2020.786

Recepción: 12 mayo 2020

Aprobación: 25 Agosto 2020

Publicación: 4 Septiembre 2020

\section{RESUMEN}

Se reporta un caso de un semental porcino de la raza landrace, de 6 años y un peso de $250 \mathrm{Kg}$, el cual fue atendido por el servicio clínico ambulatorio del área de Clínica Médico-Quirúrgica de Grandes Animales de la Facultad de Medicina Veterinaria y Zootecnia de la Universidad de Córdoba. La anamnesis indica que el reproductor presentó un enrojecimiento de la región escrotal izquierda, así como una marcada asimetría entre ambos testículos con predominio del izquierdo, dolor e incomodidad en días anteriores, por lo que se le realizaron punciones con retirada de líquidos, pero con reincidencia posterior. Teniendo en cuenta la notable gravedad del evento y la disminución de la función reproductiva, así como el deterioro en la salud y el bienestar del animal, se decidió realizar una orquiectomía bilateral con resección escrotal. Durante el procedimiento quirúrgico bajo anestesia general, se evidenció al incidir la túnica albugínea y exponer por completo el testículo izquierdo, fue evidente una torsión en $720^{\circ}$ del cordón espermático, así como aumento de tamaño y color negruzco, siendo indicativo de isquemia y necrosis. Se concluye la importancia de conocer que en casos de aumento de tamaño de testículos se debe considerar la torsión como una opción diagnóstica, así como la coloración negruzca del órgano como indicativo de una isquemia completa, lo que confirma una torsión testicular de más de $6 \mathrm{Hs}$, tiempo mínimo que reporta la literatura para causar daños irreversibles en el testículo.

Palabras claves: Isquemia; porcino; orquitis; orquiectomía.

\footnotetext{
ABSTRACT

A case of a pig stallion of the landrace breed, 6 years old and weighing $250 \mathrm{~kg}$, is reported, which was attended by the outpatient clinical service of the Large Animal Medical-Surgical Clinic area of the Faculty of Veterinary Medicine and Zootechnics from the University of Córdoba. The anamnesis indicates that the player presented redness in the left scrotal region, as well as a marked asymmetry between both testicles with a predominance of the left, pain and discomfort in

Como citar (Vancouver).

Reyes-Bossa BJ, Henriquez-Crespo RJ, Cardona-Álvarez JÁ. Torsión del cordón espermático en un semental porcino. Rev Colombiana Cienc Anim. Recia. 2020; 12(2):e786. https://doi.org/10.24188/recia.v12.n2.2020.786
} 
previous days, for which punctures were performed with fluid withdrawal, but with recurrence later. Taking into account the remarkable severity of the event and the decrease in reproductive function, as well as the deterioration in the health and well-being of the animal, it was decided to perform a bilateral orchiectomy with scrotal resection. During the surgical procedure under general anesthesia, when the tunica albuginea was incised and the left testicle was completely exposed, a $720^{\circ}$ torsion of the spermatic cord was evident, as well as an increase in size and blackish color, indicating ischemia and necrosis. The importance of knowing that in cases of testicular enlargement, torsion should be considered as a diagnostic option is concluded, as well as the blackish coloration of the organ as indicative of complete ischemia, which confirms a testicular torsion of more than $6 \mathrm{Hs}$, the minimum time reported in the literature to cause irreversible damage to the testicle.

Keywords: Ischemia; porcine; orchitic; orchiectomy.

\section{INTRODUCCIÓN}

La torsión del cordón espermático o vólvulos del cordón espermático es una emergencia quirúrgica, ya que una lesión isquémica de más de 4 horas amenaza seriamente la viabilidad continúa del testículo ipsilateral (1). La torsión intravaginal, es causada por una malformación congénita del proceso vaginal. En esta malformación, la túnica vaginal no solo cubre el testículo y el epidídimo, sino también el cordón espermático. Esto crea una "deformidad de campana" que permite que el testículo Gire libremente dentro de la túnica vaginal (2).

Esta afección se caracteriza principalmente por dolor escrotal, edema y enrojecimiento. Existen algunos factores relacionados con la torsión como lo son: el aumento del volumen testicular, tumores testiculares, testículo con posición horizontal, historia de criptorquidismo, cordón espermático con porción intra escrotal larga, inserción alta o proximal de la túnica vaginalis al cordón espermático, traumatismo y ejercicio reciente. También existen factores ambientales que se han asociado con la torsión testicular como la temperatura baja (3).

Usualmente las torsiones ocurren en ausencia de cualquier evento precipitante; En humanos se ha reportado que solo 4 a 8 por ciento de los casos son resultado de traumas (2). La supervivencia del testículo afectado depende de un rápido diagnóstico y la corrección de la torsión (4).

A pesar del diagnóstico rápido y de la orquiectomía o la preservación del testículo afectado, la infertilidad sigue siendo una secuela importante de la torsión testicular. Por lo tanto, se cree que la torsión testicular unilateral da como resultado una lesión testicular contralateral. Estudios en humanos y animales han demostrado defectos en la espermatogénesis tras un evento de torsión (5).

El testículo contralateral generalmente es considerado como adecuado para el mantenimiento de la función hormonal y reproductiva del animal. Aunque ya se sabe que la lesión isquémica, traumática o térmica unilateral causan Lesiones que afectan la espermatogénesis en el testículo contralateral (6).

Por otra parte, la autoinmunización, algunos episodios subclínicos de torsión testicular contralateral, liberación de enzimas acrosómicas, respuesta neuroendocrina o vasomotora apareada durante la torsión, y la presencia de defectos subyacentes en la espermatogénesis han sido culpadas por el daño testicular contralateral (5).

El diagnóstico clínico de la torsión del cordón espermático se basa en un historial de aparición repentina de dolor unilateral severo e hinchazón escrotal, En ausencia de una historia típica, la diferenciación de torsión aguda de una epididimitis es difícil y. en la práctica, los dos son indistinguibles en el 50\% de los casos así lo afirman estudios en humanos. Algunas ayudas de diagnóstico que son útiles para diferenciar la torsión de la epididimitis aguda incluyen la gammagrafía, la ecografía Doppler y la ecografía en escala de grises (7).

Luego de la detorsión del testículo, el aumento gradual después de una disminución en el flujo sanguíneo puede llevar a un daño adicional en los tejidos a través de un aumento de la peroxidación lipídica después de la producción de radicales libres de oxígeno como se muestra en otros órganos. Este fenómeno se denomina "lesión por reoxigenación post-hipóxica" o más comúnmente llamada "lesión por reperfusión" (8).

Diferentes tratamientos se han reportado para prevenir o aminorar la lesión por reperfusion y la isquemia en los casos de torsión testicular. Inhibidores de la fosfodiesterasa tipo 5 (PDE5) como el sildenafil es uno de los fármacos que han sido estudiados para dicho propósito (9). También ha sido evaluado el efecto de un agonista alfa 2 adrenérgico 
altamente selectivo como lo es la dexmedetomidina, para el cual se tuvieron resultados muy positivos y que podría ser usado para atenuar dichos efectos luego de la detorsión (10). Por último, un inhibidor selectivo de la ciclooxigenasa 2 (COX2) como la nimesulida, demostró efectividad para aminorar los daños post reperfusion (11). Siendo estos fármacos opciones terapéuticas para aquellos animales donde se realice detorsión del testículo afectado, y así diseñar protocolos encaminados a una completa recuperación de animales con torsión testicular.

El objetivo del presente estudio es presentar el primer reporte de caso de una torsión de cordón espermático en un cerdo reproductor en el departamento de Córdoba, realizar una breve revisión de lo que esta patología traduce y mencionar los medicamentos que pueden ser utilizados para atenuar los efectos de lesión por reperfusion en animales que sean tratados quirúrgicamente.

\section{MATERIALES Y MÉTODOS}

Sitio de estudio. Fue atendido por el servicio de Clínica Ambulatoria de Grandes Animales de la Universidad de Córdoba, un semental porcino de la raza Landrace, con una edad de 6 años y un peso de $250 \mathrm{Kg}$, ubicado en la Universidad de Córdoba sede Berastegui en el Municipio de Ciénega de Oro, Córdoba.

El personal encargado de la producción reporto un enrojecimiento de la región escrotal izquierda, así como una marcada asimetría entre ambos testículos con predominio del izquierdo, dolor e incomodidad en días anteriores. Además, fue primeramente diagnosticado como hidrocele y le fueron realizadas varias punciones con agujas de 18G para realizar drenajes del líquido, siendo insatisfactorio dicho procedimiento, por lo que días después reincidía el aumento de volumen y cada vez empeoraba el estado general del animal

Examen Físico. Se realiza evaluación clínica al momento de llegada, el cual presenta, a la inspección asimetría con aumento de volumen del testículo izquierdo, así como áreas de eritema focalizado (Figura 1), de igual forma, a la palpación se determinó presencia de dolor e hipotermia en el testículo izquierdo.

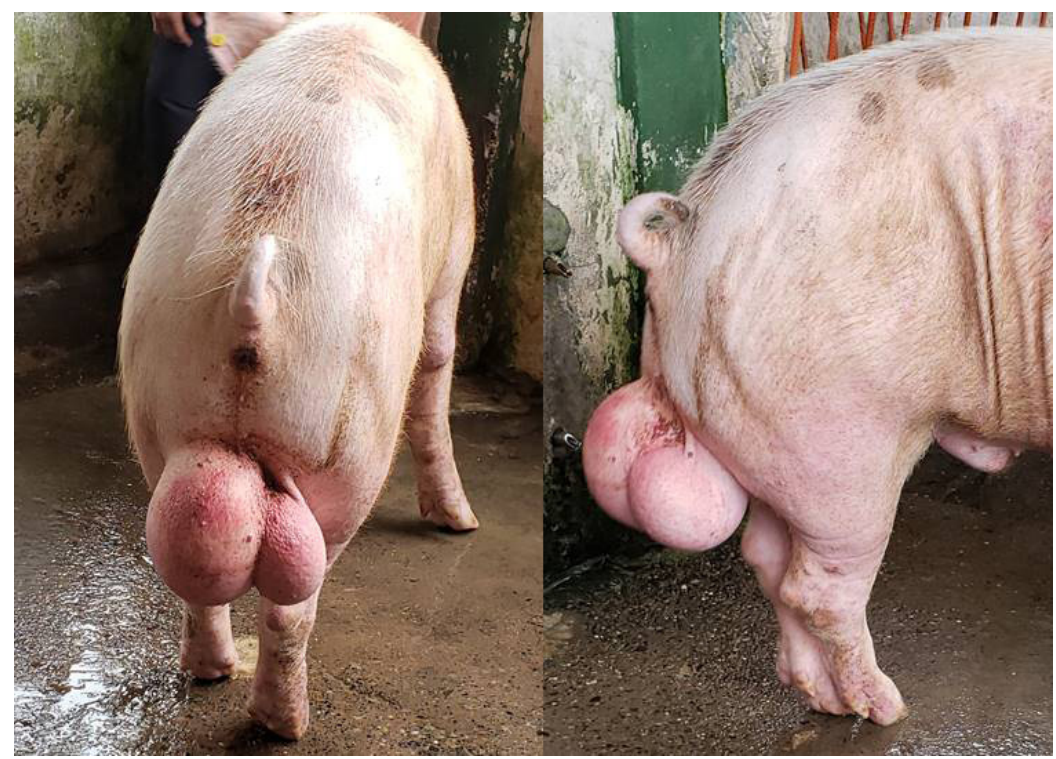

Figura 1. Asimetría testicular. Observe el marcado aumento de volumen del testículo izquierdo en comparación con el testículo derecho en vistas posterior y lateral respectivamente.

Procedimiento. Teniendo en cuenta la notable gravedad del evento y la disminución de la función reproductiva, así como el deterioro en la salud y el bienestar del animal, se decidió realizar una orquiectomia bilateral con resección escrotal.

Para realizar el procedimiento quirúrgico en el animal se tuvieron en cuenta las normas técnicas referentes a los principios éticos internacionales para la investigación biomédica con animales del CIOMS (Council for International Organizations of Medical Sciences) establecida por la UNESCO (United Nations Educational, Scientific and Cultural Organization) y la OMS (Organización Mundial de la Salud) de 1949 y de la Ley 84 de Octubre 27 de 1989 (Estatuto Colombiano de Protección Animal) (12). 
Protocolo Anestésico. Todo el procedimiento quirúrgico fue realizado bajo anestesia general, Como premeditación se utilizó xilacina (Xilacina 10\%, Erma, Colombia) a dosis de $1 \mathrm{mg}$ x Kg Vía intramuscular. Como inductor se utilizó una mezcla de Azaperona (Stresnil, Janssen, USA) a dosis de $3 \mathrm{mg}$ x Kg + Ketamina (Kentatir 5\%, Over, Colombia) a dosis de 10 $\mathrm{mg} \times \mathrm{kg}$. Como analgésico en el post operatorio se utilizó flunixin de meglumine (flunixin 5\%, vecol, Colombia) a dosis de 2,2 mg x Kg y como antibiótico oxitetraciclina LA (oxi LA, Erma, Colombia) a dosis de 20 mg x kg.

\section{RESULTADOS}

Hallazgos quirúrgicos. Al incidir la túnica albugínea y exponer por completo el testículo izquierdo, fue evidente una torsión en $720^{\circ}$ del cordón espermático (Figura 2), así como aumento de tamaño y color negruzco, siendo indicativo de isquemia y necrosis. El aumento de tamaño correspondía aproximadamente a tres veces el tamaño del testículo contralateral (Figura 3a), y una isquemia completa en todo el órgano, hecho que confirma una torsión testicular de más de 6 Hs (Figura 3b), tiempo mínimo que reporta la literatura para causar daños irreversibles en el testículo. Consolidándose así como el primer reporte de torsión testicular de un cerdo en el departamento de Córdoba.

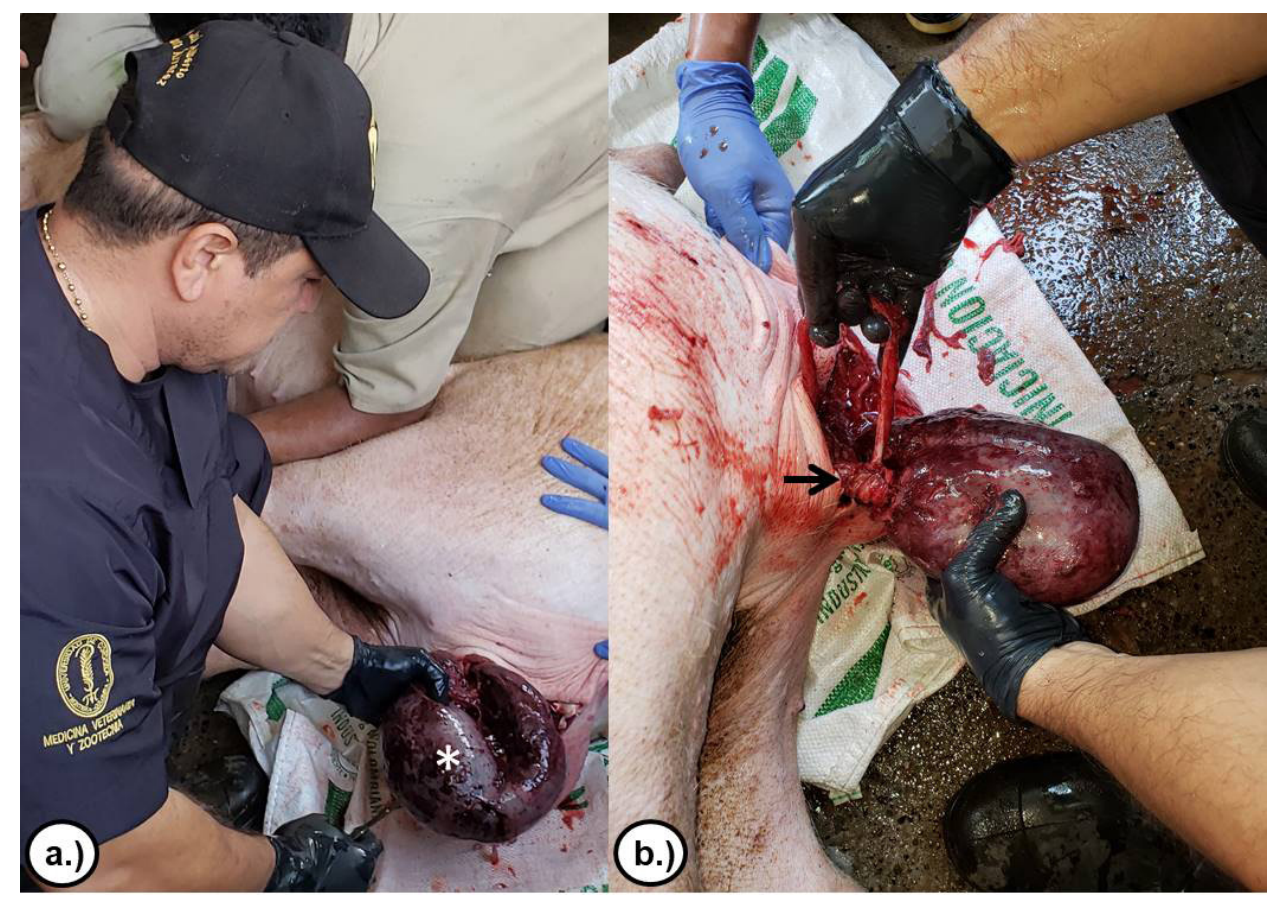

Figura 2. a.) Observe el aumento de tamaño y color negruzco (asterisco), siendo indicativo de isquemia y necrosis. b.) Note la evidente torsión en $720^{\circ}$ del cordón espermático sobre su mismo eje (flecha).

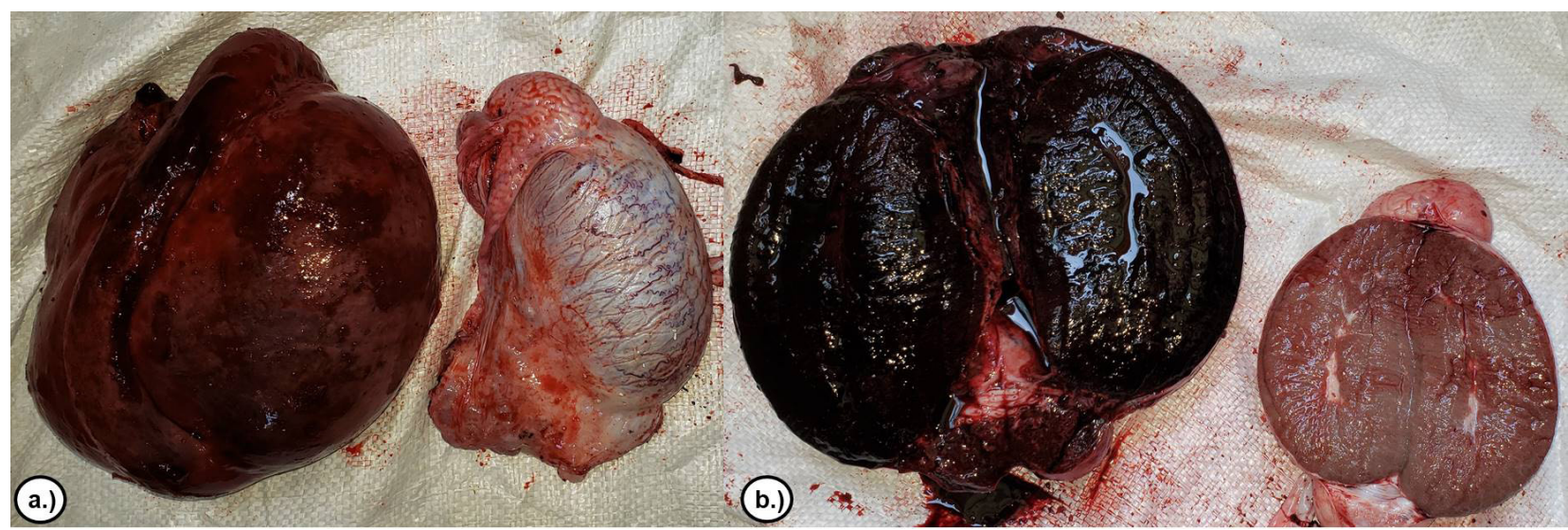

Figura 3. a.) Aumento de tamaño de aproximadamente a tres veces el tamaño del testículo contralateral. b.) Parénquima testicular isquémico y necrosado lo que confirma una torsión testicular de más de 6 Hs. 


\section{DISCUSIÓN}

Inicialmente las características de eritema y aumento de volumen confundieron a los operarios, quienes llegaron a pensar en picadura de arañas, sin embargo, según lo reportado por Cardona, 2018 (13), los accidentes ponzoñosos cursan con dermonecrósis, por lo que el caso no presento dicha característica descartándose de las posibilidades clínicas.

Son escasos los reportes que existen en la literatura científica sobre torsión testicular en cerdos. En otras especies como la humana, es más común encontrar reportes de esta afección. Por ejemplo, Anderson y Williamson, 1998 (14), realizaron un estudio retrospectivo de 670 casos de torsión testicular en humanos.

En humanos la edad promedio en la que se reportan los casos de torsión, es en adultos jóvenes de 15 a 20 años. Como es el caso del reporte de torsión testicular en un joven de 15 años que llego al Hospital Regional de Alta Especialidad Dr. Gustavo A. Rovirosa Pérez con dolor súbito y continuo en el testículo izquierdo (3). Lo cual no coincide con lo encontrado en este reporte, ya que era un animal reproductor de avanzada edad, sin embargo no se descarta que se de en adultos de edad temprana, por la pobre documentación de estos casos que no permite llegar a esta conclusión.

Si bien se ha reportado en otros animales, en la revisión de literatura del estudio no se encontró reportes en cerdos, pero si diseños experimentales para estudiar más a fondo la fisiopatología de la enfermedad, modelos de torsión experimental se reportan para conejos, cobayos y ratas $(6,9,15)$.

Visser y Heyns (1), afirman que la lesión en el testículo afectado es causada por una combinación de isquemia y lesión por reperfusión por especies reactivas de oxígeno, que surgen de la activación del sistema de la xantina oxidasa en células parenquimatosas o de leucocitos que se adhieren a las paredes de las vénulas re-perfundidas antes de someterse a la diapédesis en el tejido intersticial. Siendo acorde con lo encontrado en el animal, ya que presento signos de endotoxemia y marcada depresión.

En conclusión este es el primer reporte de una torsión del cordón espermático en un cerdo en el departamento de córdoba. Por lo tanto, es importante para los médicos veterinarios del sector, tener en cuenta esta patología como uno de los diferenciales en cualquier caso de aumento de tamaño y dolor escrotal en cerdos de cualquier producción.

\section{REFERENCIAS}

1. Visser A, Heyns C. Testicular function after torsion of the spermatic cord. BJU International. 2003; 92: 200-203. https://doi.org/10.1046/j.1464-410X.2003.04307.x

2. Ringdahl E, Teague L. Testicular Torsion. Am Fam Physician. 2006; 74(10):1739-1743. https://www.aafp.org/ afp/2006/1115/p1739.html

3. García G, Bravo A, Bautista R. Torsión testicular: reporte de un caso. Cirugía y Cirujanos. 2017; 85:432-435. https:// doi.org/10.1016/j.circir.2016.05.014

4. Turner T. Acute Experimental testicular Torsion. Journal of Andrology. 1985; 6(1):65-72. https://doi. org/10.1002/j.1939-4640.1985.tb00817.x

5. Lievano G, Nguyen L, Radhakrishnan J, Fornell L, John E. New Animal Model to Evaluate Testicular Blood Flow During Testicular Torsion. Journal of Pediatric Surgery. 1999; 34(6):1004-1006. https://doi.org/10.1016/S0022$\underline{3468(99) 90778-9}$

6. Chakraborty J, Jhunjhunwala L, Nelson L, Young M. Effects of Unilateral Torsion of the Spermatic Cord on the Contralateral Testis in Human and Guinea Pig. Archives of Andrology. 1980; 4(2):95-108. https://doi. org/10.3109/01485018008986475

7. Hricak H, Lue T, Filly R, Alpers C, Zeineh S, Tanagho E. Experimental Study of the Sonographic Diagnosis of Testicular Torsion. J Ultrasound Med. 1983; 2(8):349-356. https://doi.org/10.7863/jum.1983.2.8.349 
8. Akgur F, Kilinc K, Aktug T. Reperfusion injury after detorsion of unilateral testieular torsion. Urol Res. 1993; 21:395399. https://doi.org/10.1007/BF00300075

9. Beheshtian A. Protective effects of sildenafil administration on testicular torsion/detorsion damage in rats. World J Urol. 2008; 26:197-202. https://doi.org/10.1007/s00345-008-0243-6

10. Hanci V. Effect of Dexmedetomidine on Testicular Torsion/Detorsion Damage in Rats. Urol Int. 2010; 84:105-111. https://doi.org/10.1159/000273476

11. Polat E, Bozkurt A, Cimen F, Gulaboglu M, Altuner D. The investigation of the protective effects of nimesulide on experimental testicular ischemia-reperfusion injury in rats. Rev. Int. Androl. 2018; 18(2):55-62. https://doi. org/10.1016/j.androl.2018.08.001

12. Mrad A. Ética en la investigación con modelos animales experimentales. Alternativas y las 3 RS de Russel. Una responsabilidad y un compromiso ético que nos compete a todos. Rev Col Bioética. 2006; 1(1):163-184. https:// www.redalyc.org/pdf/1892/189217283010.pdf

13. Cardona AJ. Atlas de Dermatología Tropical en Grandes Animales. Fondo Editorial de la Universidad de Córdoba. Universidad de Córdoba. Montería, Colombia. 2018. https://articulo.mercadolibre.com.co/MC0-578220368-atlasde-dermatologia-tropical-en-grandes-animales- JM

14. Anderson J, Williamson R. Testicular torsion in Bristol: a 25-year review. Br. J. Surg. 1988; 75(10): 988-992. https:// doi.org/10.1002/bjs.1800751015

15. Cerasaro T, Nachtsheim D, Otero F, Parsons C. The effect of testicular torsion on contralateral testis and the production of antisperm antibodies in rabbits. The Journal of Urology. 1984; 132(3):577-579. https://doi.org/10.1016/S0022$\underline{5347(17) 49750-3}$ 